\title{
LA VISITE PRE-OPERATOIRE EN CHIRURGIE CARDIO-VASCULAIRE*
}

\author{
Bernard Paiement, Jean-Guy Maillé, Marcel Boulanger, Jean Taillefer, \\ Philippe Sahab, Conrad Pelletier et Ihor Dyrda \\ RÉSUMÉ
}

Ce texte destiné aux résidents d'anesthésie-réanimation en début de stage dans un service cardio-vasculaire, discute du risque opératoire et de certains problèmes courants chez les patients devant subir une intervention chirurgicale cardiaque ou vasculaire. On classifie le risque en risque habituel, accru ou élevé, selon la présence de facteurs aggravants du risque (mauvaise fonction ventriculaire, angor instable, âge, etc. ...). On propose une évaluation du risque habituel pour certaines interventions courantes. Parmi les problèmes courants rencontrés chez ces malades, on retrouve entre autres des pathologies pulmonaires, des problèmes de coagulation, le diabète et l'insuffisance rénale. La prémédication utilisée chęz ce type de malade et l'approche adoptée à l'Institut de Cardiologie de Montréal y sont discutées.

LES BUTS DE LA VISITE pré-opératoire sont les mèmes en chirurgie cardiovasculaire que chez tous les autres opérés, à savoir:

1. Prise de connaissance du dossier et évaluation du risque.

2. Recherche des problèmes particuliers méritant une attention spéciale avant de procéder à l'anesthésie et à la chirurgie (problèmes respiratoires, de coagulation, etc.).

3. Etablissement d'un climat de confiance et de collaboration avec le patient.

4. Prescription de la prémédication.

\section{LE RISQUE OPERATOIRE}

Lorsque l'on parle de risque, on est porté à penser en termes de mortalité péri-opérataire en rapport avec une pathologie donnée. Il faut également s'habituer à penser en termes de morbidité. Le patient qui quitte l'hôpital hémiplégique après une intervention n'apparaît pas dans les statistiques de mortalité, mais son résultat n'en est pas moins catastrophique. La morbidité transitoire (respiratoire, rénale, infec-

Bernard Paiement, M.D., F.R.C.P.(C); Jean-Guy Maillé, M.D., F.R.C.P.(C); Marcel Boulanger, M.D., F.R.C.P.(C); Jean Taillefer, M.D., F.R.C.P.(C); Philippe Sahab, M.D., Conrad Pelletier, M.D., F.R.C.S.(c), F.A.C.S. et thor Dyrda, M.D., F.R.C.P.(C), F.A.C.C. Départements d'AnesthésieRéanimation, de Chirurgie et de Médecine, Institut de Cardiologie de Montréal et Université de Montréal, Montréal, Québec, Canada.

* Texte destiné aux résidents d'AnesthésieRéanimation en stage à l'Instituı de Cardiologie de Montréal.

Adresse pour tirés-à-part: B. Paiement, M.D., Département d'Anesthésie-Réanimation, Institut de Cardiologie de Montréal, 5000 est, rue Bélanger, Montréal, Québec, Canada HIT IC8. 584 tieuse, etc.) est également importante. Ses conséquences en souffrance et en séjour hospitalier prolongé justifient tous les efforts de prévention reconnus comme efficaces.

Le risque opératoire - mortalité et morbidité doit donc être considéré dans l'optique de la gravité de la maladie, de son pronostic si elle n'est pas traitée chirurgicalement, et des bénéfices éventuels de la chirurgie. Deux classifications d'usage courant contribuent à évaluer la gravité d'une cardiopathie donnée, à savoir la classification de l'incapacité de la New York Heart Association, et la classification canadienne de l'angine.

Le tableau I rappelle ces deux classifications. Il rappelle également la classification de l'état physique de l'American Society of Anesthesiologists (ASA).

\section{Classification de l'état physique de l'ASA}

On dit que la classification de l'ASA décrit un état physique et non un risque anesthésique. Cela est vrai. Il existe toutefois une corrélation bien établie entre la mortalité post-opératoire (précoce et tardive) et la classification du malade selon les critères de l'ASA. La classification de l'ASA est le meilleur prédicteur de la mortalité par causes non cardiaques et un bon prédicteur de la mortalité de causes cardiaques.'

\section{Notion de risque habituel}

On ne peut jamais parler d'un risque "zéro", même pour un cas très mineur. De même, on ne peut pas dire qu'un risque de mortalité d'un ou de deux pour cent soit un bon risque en chirurgie cardiaque si on le compare au risque d'une réparation de hernie. ${ }^{2}$ Nous préférons parler de risque habituel, à distinguer du risque moyen d'une in- 
TABLEAU I

\section{INCAPACITE CLASSIFICATION DE LA NYHA}

Classe I Pas de limitation de l'activité physique. Les activités ordinaires ne causent pas de fatigue, de palpitations, de dyspnée ou d'angine

Classe II Confortable au repos. Symptomatique à l'activité physique ordinaire.

Classe III Limitation marquée à l'activité. Symptômes avec effort moindre que l'activité ordinaire

Classe IV Symptômes au repos

\section{ANGINE CLASSIFICATION CANADIENNE}

Classe $0 \quad$ Pas d'angine à l'effort maximal ou spontané

Classe 1 Pas d'angine à l'activité normale (escaliers, marche ra pide, paquets). Angine à l'effort violent ou prolongé - sport

Classe Il Doit modérer l'activité normale.

Angine: à la marche rapide

à la marche en côte, au vent, au froid

à la montée rapide d'un escalier

Classe III Limitation à l'activité ordinaire.

Angine: à la montée d'un escalier à pas normal

à la marche (un ou deux coins) en terrain plat

Classe IV Angine au moindre effort: se raser, toilette

quelques pas

au repos

Classification de l'etat physigue de l'asa

Classe I Sujet normal en bonne santé

Classe 11 Sujet porteur d'une affection systémique légère

Classe IIl Sujet atteint d'une affection systémique grave qui limite son activité, sans le rendre invalide

Classe IV Sujet atteint d'une affection systemique incapacitante qui est un danger constant pour sa vie

Classe V Moribond que l'on s'attend de voir mourir dans les 24 heures, avec ou sans chirurgie (sauvetage)

En cas d'urgence, on ajoute la lettre $E$ devant la classe, ce qui élève le risque de cette classe.

tervention donnée. Le risque habituel se réfêre aux patients ne présentant pas certains facteurs connus d'aggravation du risque. Ainsi, on peut parler d'un risque habituel de mortalité d'un ou de deux pour cent dans le cas d'un pontage aorto-coronarien pour un malade en bon état général et avec une fonction ventriculaire normale. La même intervention effectuée chez un homme de 72 ans comporte un risque plus grand. Le risque habituel peut être bas (fermeture de CIA), modéré (remplacement mitral) ou élevé (remplacement multivalvulaire, anévrysme abdominal rupturé).

Le risque habituel est établi à partir du risque moyen, lequel inclut des patients à risque bas et des malades à risque élevé. Le tableau II tente d'évaluer le risque habituel d'actes opératoires courants en chirurgie cardio-vasculaire. Cette liste est difficile à établir à cause de la variation des résultats d'un milieu à l'autre, et du fait que la plupart des statistiques publiées sont des valeurs moyennes se rapportant à l'ensemble des patients opérés pour une pathologie dans une institution donnée. Le tableau III en annexe décrit la mortalité hospitalière chirurgicale dans notre institution au cours de la dernière année administrative (avril 1979-avril 1980).

\section{Facteurs aggravants du risque}

Neuf facteurs principaux contribuent à élever la mortalité (et la morbidité) des patients en chirurgie cardio-vasculaire (tableau IV):

\section{Mauvaise fonction ventriculaire}

L'état du muscle cardiaque est un facteur important du risque opératoire. La meilleure façon de l'évaluer est la ventriculographie que l'on effectue en salle hémodynamique (ciné-ventriculographie) ou de façon non invasive en médecine nucléaire. Les images obtenues permettent d'évaluer la qualité de la contraction et d'établir la fraction d'éjection.

(a) Zones akinétiques ou hypokinétiques

La présence et, surtout, l'étendue des zones 
TABLEAU II

Chirurgie cardio-vasculaire risQue habituel

\begin{tabular}{|c|c|}
\hline Chirurgie cardiaque & $\begin{array}{l}\text { Risque de } \\
\text { mortalité } \\
(\%)\end{array}$ \\
\hline $\begin{array}{l}\text { Fermeture de ClA ou de CrV } \\
\text { Correction de canal artériel ou de coarctation } \\
\text { de l'aorte }\end{array}$ & $<1$ \\
\hline $\begin{array}{l}\text { Commissurotomie mitrale } \\
\text { Pontage aorto-coronarien }\end{array}$ & $<2$ \\
\hline $\begin{array}{l}\text { Résection d'anévrysme du ventricule gauche } \\
\text { (avec ou sans pontage) } \\
\text { Remplacement de valve mitrale ou aortique }\end{array}$ & $<5$ \\
\hline Remplacement de valves mitrale et aortique & $8-10$ \\
\hline $\begin{array}{l}\text { Remplacement mitral et plastie tricuspidienne } \\
\text { Remplacement mitral ou aortique et PAC }\end{array}$ & $10-15$ \\
\hline $\begin{array}{l}\text { Chirurgie vasculaire } \\
\text { Chirurgie des carotides ou des extrémités } \\
\text { Pontage aorto-iliaque ou aorto-fémoral }\end{array}$ & $<1$ \\
\hline $\begin{array}{l}\text { Résection d'anévrysme de l'aorte abdominale } \\
\text { opéré de facon élective }\end{array}$ & $<5$ \\
\hline Anévrysme de l'aarte abdominale rupturé & 50 \\
\hline Aorte thoracique & 15 \\
\hline
\end{tabular}

TABLEAU III

Institut de Cardiologie de Montréal Mortalité hospitaliere chirurgicale (avril 1979 à avril 1980)

\begin{tabular}{lcc}
\hline & Décès/Patients & $\%$ \\
\hline Chirurgie congénitale & $0 / 26$ & 0 \\
Chirurgie coronarienne & & \\
Pontages isolés & $8 / 483$ & 1.7 \\
Anévrysmes du ventricule gauche avec ou sans pontages & $2 / 42$ & 4.8 \\
$\quad$ Total & $10 / 525$ & 1.9 \\
Chirurgie valuulaire & & \\
Commissurotomies ou plasties mitrales & $0 / 15$ & 0 \\
Remplacement valvulaire aortique & $2 / 45$ & 4.4 \\
Remplacement valvulaire mitral & $4 / 89$ & 4.5 \\
Remplacement mitral et aortique & $3 / 33$ & 9.4 \\
Remplacement mitral et plastie tricuspidienne & $2 / 14$ & 14.3 \\
Remplacement mitral et PAC & $3 / 20$ & 15 \\
Remplacement aortique et PAC & $2 / 16$ & 12.5 \\
Remplacement aortique, PAC, et résection d'anévrysme & & 14.1 \\
$\quad$ de l'aorte ascendante & $1 / 7$ & 7.1 \\
Total & $17 / 239$ & 3.4 \\
Chirurgie à cazur-ouvert - Tolal & $27 / 790$ & 5.8 \\
Chirurgic vasculaire & $7 / 122$ & .5 \\
Pacemakers & $1 / 221$ & 3 \\
Grand roral & $35 / 1133$ & \\
\hline
\end{tabular}

d'hypo ou d'akinésie permettent de juger de la qualité du myocarde. On peut cependant l'évaluer de façon plus précise en faisant le calcul de la:

(b) Fraction d'éjection, qui est le rapport:

Volume Ventriculaire en Fin de Diastole - Volume en Fin de Systole

Volume en Fin de Diastole
Une valeur supérieure à 0.55 est considérée normale, alors qu'un rapport entre 0.4 et 0.55 , fréquent après un infarctus du myocarde non compliqué et sans insuffisance cardiaque, est considéré acceptable. Une fraction inférieure à 0.30 au repos correspond à une mauvaise fonction ventriculaire.

On peut également porter un jugement de mauvaise fonction ventriculaire lorsque la pres- 
TABLEAU IV

Chirurgie cardio-vasculatre

Facteurs d'aggravation du risque chirurgical

1. Mauvaise fonction ventriculaire

2. Angor instable ou infarctus récent

3. Insuffisance cardiaque

4. Autre atteinte importante non stabilisée ou non corrigible

5. Age avancé

6. Réintervention

7. Obésité importante

8. Urgence ou chirurgie de sauvetage

9. Chirurgie non cardiaque en présence de cardiopathie importante.

sion télédiastolique ventriculaire gauche au repos est supérieure à $18 \mathrm{mmHg}(2.39 \mathrm{kPa})$ ou à $25 \mathrm{mmHg}(3.33 \mathrm{kPa})$ après la ventriculographie. Cette dysfonction peut être temporaire et réversible dans le cas du coronarien en angine instable dont le myocarde est hypoxique et non compliant. Chez le porteur d'une sténose aortique et chez l'hypertendu, l'élévation de la pression télédiastolique ventriculaire gauche reflète une diminution de la compliance du muscle plutôt qu'une mauvaise fonction ventriculaire.

\section{Angor instable ou infarctus récent}

On doit de temps à autre procéder à une chirurgie coronarienne chez des malades en angine instable qui demeurent symptomatiques malgré une thérapie agressive (vasodilatateurs en perfusion, bêta-bloquants à haute dose ou en perfusion) ou encore chez des patients ayant subi récemment un infarctus. Il va de soi que le risque est alors plus élevé. Une étude récente effectuée dans notre institution ${ }^{3}$ a confirmé les rapports déjà publiés, à savoir que l'incidence d'infarctus périopératoire est plus élevée chez les malades ayant présenté un infarctus moins de six mois auparavant

\section{Insuffisance cardiaque non compensée}

La persistance des signes cliniques d'insuffisance cardiaque (œdème, râles sous-crépitants aux bases pulmonaires, dyspnée nocturne paroxystique, orthopnée, un $B_{3}$ ) au moment de la chirurgie se traduit par un risque plus élevé.

4. Autre atteinte importante non stabilisée ou non corrigible

Nous classifions sous ce titre-tiroir plusieurs facteurs qui aggravent le risque habituel.

Correction complète des lésions impossible (atteinte sévère des trois troncs coronariens et une seule coronaire pontable).
Atteinte respiratoire sévère; insuffisance rénale ou hépatique significative.

Une endocardite évolutive.

Une hypertension artérielle sévère non contrôlée ou non contrôlable.

Une histoire d'arythmies importantes (salves d'extrasystoles ventriculaires - épisodes de tachycardies supraventriculaires, etc.)

Un diabète non contrôlé.

Un mauvais état nutritionnel, comme la cachexie cardiaque que l'on retrouve chez les mitraux à un stade avancé de la maladie.

Une hémiparésie ou une hémiplégie, etc.

Nous reverrons certains de ces facteurs en discutant de problèmes particuliers.

\section{Age}

Dans son étude sur les facteurs de complications graves et de morts cardiaques dans les cas de chirurgie non cardiaque, Goldman ${ }^{4}$ a retenu l'âge avancé ( $>70$ ans) parmi les facteurs importants de morbidité et de mortalité. La même situation se retrouve en chirurgie cardiaque: audessus de 65 ans, le risque s'élève de façon significative. Une étude des patients de plus de 65 ans ayant subi un pontage aorto-coronarien dans notre institution depuis l'ère de la cardioplégie, ${ }^{5}$ a montré une mortalité de cinq pour cent (le risque habituel des pontages est de un à deux pour cent).

\section{Réintervention}

La mortalité et la morbidité sont plus élevées en chirurgie cardio-vasculaire lors des réinterventions parce que:

1. La dissection est souvent plus difficile, ce qui allonge la durée de l'intervention.

2. Il existe un risque de traumatisme (ex: déchirure du ventricule droit lors de la sternotomie).

3. Le saignement post-opératoire est plus important que lors d'une première intervention.

4. Le danger d'infection est plus grand.

\section{L'obésité importante}

Le risque de mortalité et de complications post-opératoires des malades qui présentent une obésité modérée (inférieure à 30 pour cent) est à peu près du même ordre que celui de la population normale alors qu'il s'élève de façon significative chez les grands obèses. ${ }^{6}$ Ceux-ci présentent plus souvent des complications respiratoires, tolèrent mal la position couchée et sont sujets à l'hypoxémie post-opératoire. L'incidence d'infarctus du myocarde, de thrombophlébites et d'embolies pulmonaires, ainsi que d'infections de plaies est plus élevée chez eux. 
Une formule simple a été élaborée ${ }^{3}$ à partir de l'index de masse corporelle ${ }^{6}$ et permet d'identifier les obésités significatives. Il suffit de soustraire de la taille exprimée en centimètres le poids en kilos. Si la différence de ces deux chiffres est de 100 ou plus, il n'y a pas d'obésité. Une différence d'environ 90 signale une obésité modérée, alors que l'obésité est importante lorsque la différence est de 82 ou moins. Par exemple, on peut facilement se représenter qu'un individu qui mesure $175 \mathrm{~cm}$ et pèse $70 \mathrm{~kg}$ n'est pas obèse (différence: 105); si le mème individu gagne $25 \mathrm{~kg}$, il le devriendra de façon significative (175 $-95=80$ )

\section{Urgence-Chirurgie de sauverage}

Cet autre facteur aggravant qu'avait retenu Goldman dans son étude du risque dans la chirurgie non cardiaque, ${ }^{4}$ est également vrai en chirurgie cardio-vasculaire.

En pratique, nous opérons en urgence ou en semi-urgence:

Certains valvulaires décompensés dont l'état s'aggrave malgré le traitement médical.

Les coronariens en angine instable malgré un traitement médical agressif.

Les patients qui présentent une complication à la coronarographie.

Les cas d'ischémie aiguë des membres.

II n'y a pas d'indication à opérer en urgence un patient en infarctus aigu êvolutif. La mortalité hospitalière de l'infarctus aigu? est moins êlevée que la mortalité de la chirurgie de pontage aortocoronarien effectuée en urgence dans le but de diminuer la zone infarcie. ${ }^{\mathrm{B}}$

9. Chirurgie non-cardiaque en présence d'une cardiopathie importante ou d'un infarctus récent

La meilleure façon d'illustrer ce point est au moyen d'exemples:

(a) Angioplastie carotidienne chez un malade porteur de sténoses coronaires sévères et non corrigées. Chez le coronarien symptomatique avec des lésions importantes (sténose serrée du tronc commun de la coronaire gauche), il est parfois préférable d'effectuer la chirurgie coronarienne et carotidienne en un seul temps opératoire, la carotide étant opérée avant d'aller sous CEC pour effectuer les pontages coronariens.

(b) Cholécystite aiguë chez un patient porteur d'une sténose aortique serrée et symptomatique.

(c) Ulcus perforé, chez un patient en infarctus évolutif. (d) Le coronarien ayant subi un pontage aorto-coronarien et stable est considéré à toutes fins pratiques comme un patient porteur d'un infarctus de plus de six mois. Les mêmes précautions applicables à ces patients seront indiquées chez lui.

\section{Classification du risque}

La présence ou l'absence de facteurs aggravants permet de classifier le risque en risque habituel, risque accru, et risque élevé.

Les facteurs aggravants n'ont pas tous la même importance relative, mais de façon générale, l'individu qui présente un seul facteur aggravant est considéré comme porteur d'un risque accru, alors que la présence de deux facteurs aggravants (ou plus) se traduit par un risque élevé. Lorsque le risque habituel est lui-même élevé (double remplacement valvulaire avec pontage), le risque est classifié comme tel.

\section{PROBLEMES COURANTS}

\section{Pathologies pulmonaires}

Une épreuve sommaire de la fonction respiratoire est effectuée avant l'intervention chez nos malades. Le test utilisé est celui de la boucle débit-volume (Flow-Volume Loop), ${ }^{9}$ un examen court (moins de cing minutes avec les explications) et non fatigant pour le cardiaque. On en retire plusieurs renseignements utiles tels la capacité vitale, la $\mathrm{FEV}$, et les débits expiratoires des grosses, moyennes et petites bronches. L'allure générale de la courbe permet d'identifier d'un coup d'ceil une dysfonction importante. Dans de tels cas, les mesures spirométriques habituelles sont effectuées (volumes et capacités standards). L'inhalothérapeute qui administre le test profite de la présence du patient pour lui enseigner l'utilisation de la spirométrie incitative* dont l'usage est routinier après chirurgie dans notre institution.

Il faut garder à l'esprit que chez les cardiaques avec une atteinte du coeur gauche et une pression auriculaire gauche élevée, en particulier chez les mitraux, la dysfonction pulmonaire peut être le reflet de l'hypertension vasculaire pulmonaire. Une pression veineuse pulmonaire élevée de façon chronique se traduit par un odème interstitiel pulmonaire avec ou sans odème alvéolaire et, éventuellement, par de la fibrose interstitielle. L'augmentation de l'eau extravas-

*Incentive Spirometer (Bartlett-Edwards). 
culaire pulmonaire et la fibrose interstitielle amènent une diminution de la capacité pulmonaire totale, de la capacité vitale et de la compliance pulmonaire. La capacité pulmonaire et la capacité vitale peuvent de plus être diminuées par la présence d'un gros cour' ou d'un épanchement pleural. La congestion de la muqueuse bronchique, lorsque la pression veineuse pulmonaire est élevée, explique le syndrome obstructif observé chez les patients, en plus du syndrome restrictif.

On rencontre fréquemment des malades présentant des maladies pulmonaires obstructives chroniques (MPOC). L'expérience clinique, ici comme ailleurs, a démontré que:

(a) Même avec des tests de fonction pulmonaire perturbés de façon marquée, une forte proportion de ces patients connaît une évolution pulmonaire post-opératoire semblable à celle des sujets normaux.

(b) L'utilité d'une préparation spéciale est difficile à démontrer lorsqu'il n'y a pas de recrudescence de la maladie ou de phénomènes de bronchoconstriction.

Done, nous retardons l'intervention et préparons (physiothérapie, drainage postural, antibiotiques et bronchodilatateurs si indiqué), les patients qui présentent une sur-infection récente non guérie, des sécrétions purulentes ou des phénomènes de bronchoconstriction.

Nous demandons une consultation à la physiothérapie pour enseignement des exercices respiratoires chez les grands obèses, les mitraux cachectiques et les emphysémateux symptomatiques. Lorsqu'un malade ne comprend ni le français ni l'anglais, nous tentons avec le département du nursing d'organiser un rendezvous entre le physiothérapeute et la malade en présence d'un traducteur qui puisse transmettre l'enseignement au patient

\section{Problèmes de coagulation}

Une partie de nos patients reçoivent des anticoagulants, en particulier les porteurs de prothèses valvulaires et les mitraux en fibrillation auriculaire qui ont déjà présenté des accidents emboliques. Si la chirurgie envisagée est d'ordre mineur (mise en place d'un pacemaker transveineux), il est préférable de ne pas renverser entièrement les effets des anticoagulants. Un temps de prothrombine entre 40 à 50 pour cent est considéré comme adéquat. En cas de chirurgie majeure ou traumatique (extractions dentaires multiples), il vaut mieux retrouver une coagulation normale, ce qui survient de deux à quatre jours après l'arrêt des anticoagulants. Si le temps de prothrombine est inférieur à 40 pour cent la veille de l'intervention, une dose de vitamine $\mathrm{K}_{2}$ (Aquamephyton) permettra en quelques heures de retrouver des valeurs de prothrombine normales (mais la coagulation demeure anormale pendant 48 à 72 heures). II arrive que les anticoagulants oraux soient cessés quelques jours avant une intervention et remplacés par l'héparine administrée par voie intraveineuse aux quatre heures, la dernière dose étant administrée quatre heures avant le début de l'intervention.

De nombreux médicaments affectent la fonction plaquettaire, en particulier l'acide acétyl salycilique. Une seule dose de $300 \mathrm{mg}$ d'aspirine est suffisante pour perturber l'activité plaquettaire pendant sept à dix jours. De nombreux malades nous arrivent pour une chirurgie prévue à une date prédéterminée. Or, il est difficile de contrôler la médication du malade en dehors de l'hôpital. Aussi en retrouve-t-on quelques uns qui ont pris de l'aspirine quelques jours avant leur admission à l'hôpital. Notre consultant en hématologie recommande d'attendre au moins cinq jours avant la chirurgie. Cette règle devrait être observée, en particulier dans le cas des réinterventions. Lorsque des concentrés plaquettaires sont nécessaires, une unité de plaquettes par $10 \mathrm{~kg}$ est requise pour remonter les plaquettes sanguines de 50,000 .

\section{Diabète}

Les conséquences vasculaires du diabète sont bien connues. On rencontre fréquemment cette pathologie chez les coronariens. La forme de diabète rencontrée couramment est celle de l'aduite. On sait que 80 pour cent de ces diabétiques sont des obèses, que leur glycémie est souvent contrôlable par une simple diète, que les hypoglycémiants oraux sont le plus souvent efficaces chez eux, et que ces malades sont peu susceptibles de développer un coma hyperglycémique acido-cétosique. ${ }^{10}$

On dit que si l'on ajoute le nombre d'années d'évolution de sa maladie à l'âge chronologique d'un diabétique, on a une idée de son àge physiologique." Ainsi, un opéré de 60 ans reconnu comme diabétique depuis 15 ans, peut être considéré comme un cas de gériatrie. Outre les effets du diabète sur l'évolution de la pathologie cardio-vasculaire, on devrait penser aux effets à long terme du diabète avec ses implications anesthésiques et chirurgicales, en particulier 
ceux sur le système nerveux autonome ${ }^{12}$ et sur le rein.

\section{Notions utiles}

(a) L'hypoglycémiant oral le plus utilisé en clinique, la chlorpropamide (Diabénèse) possède une durée d'action prolongée (demi-vie: 35 heures).

(b) La plus grande partie de l'insuline dissoute dans un soluté s'accole aux parois de verre (ou de plastique) du contenant, ainsi qu'à la tubulure du soluté. L'addition d'albumine à 0.1 pour cent prévient ce phénomène. Cinq millilitre du sang du patient ajoutés à un soluté fourniraient suffisamment d'albumine pour atteindre cette concentration. ${ }^{13}$

(c) On peut se faire une idée de l'osmolarité à partir des résultats du $\mathrm{Na}+\mathrm{du} \mathrm{K}+$ et de la glycémie: ${ }^{14}$

$(m E q$ de $\mathrm{Na}+0 / 00+m E q$ de $\mathrm{K}+0 / 00) \times 2$

$$
+\frac{\text { glycémie en } \mathrm{mg} \%}{18}
$$

(d) Le seuil rénal du glucose est plus élevé en présence d'insuffisance rénale. ${ }^{15}$ Dans ce cas, la recherche $d u$ glucose urinaire (Clinitest) aura tendance à sous-évaluer la glycémie.

\section{Attitude devant un diabétique stable}

Les écueils à éviter chez le patient présentant un diabète de l'adulte, ${ }^{16}$ seront l'hypoglycémie et le coma hyperosmolaire sans acido-cétose. Au point de vue pratique, cela ne pose pas de grands problèmes.

La conduite anesthésique ${ }^{10.11 .16 .17}$ chez le diabétique est bien établie et dépasse le cadre de cette revue. Mentionnons seulement que dans notre pratique journalière:

1. Nous tentons d'opérer les diabétiques tôt le matin, et s'ils doivent être retardés, un soluté glucosé est mis en route sur l'étage en attendant la chirurgie.

2. A moins de recommandation spécifique du diabétologue, la dose d'hypoglycémiants ou d'insuline du matin est omise.

3. Des contrôles de glycémie et de glucase urinaire sont effectués fréquemment en cours de chirurgie et en post-opératoire.

4. C'est l'insuline régulière que nous utilisons de routine pendant et après l'opération.

\section{Insuffisance rénale}

L'insuffisance rénale aiguë post-opératoire comporte une mortalité élevée. L'un des facteurs prédisposant à cette complication est l'existence préalable d'une insuffisance rénale modérée ou grave.

Avec une clientèle formée en grande partie d'artérioscléreux présentant souvent de l'hypertension ou un diabète, il est important de rechercher les manifestations rénales de ces pathologies.

On sait que l'urée et la créatinine demeurent normales tant que la fonction rénale est de 50 pour cent de la normale. ${ }^{16}$ Une urée et, surtout, une créatinine qui s'élèvent signalent une dysfonction rénale significative. Une épreuve de clearance de la créatine ${ }^{18}$ permet de mieux évaluer la fonction urinaire, un résultat supérieur à $80 \mathrm{ml} / \mathrm{min}$ étant normal et une clearance inférieure à $25 \mathrm{ml} / \mathrm{min}$ traduisant une atteinte importante.

Chez le patient présentant une maladie valvulaire limitant le débit cardiaque de façon significative, l'élévation de l'urée et de la créatinine reflètent l'importance de la pathologie cardiaque (azotémie prérénale) et confirment la nécessité d'une correction chirurgicale si elle est possible.

La conduite à tenir chez l'insuffisant rénal dépasse le cadre de ce texte. II faudra penser surtout en termes d'anémie et de volume sanguin, de remplacement de liquides et d'électrolytes, de médicaments à élimination rénale, et du choix des antibiotiques.

\section{Bloc de branche gauche}

On sait que l'installation d'un cathéter de Swan-Ganz peut déclencher un bloc de branche droit. ${ }^{19}$ Il est donc important de s'assurer au préalable qu'il n'y a pas de bloc de branche gauche ... Devant cette situation, on décidera le plus souvent d'attendre en fin d'intervention pour installer le Swan-Ganz, si nécessaire. Avant de refermer le thorax, nos chirurgiens fixent de routine un fil temporaire de pacemaker à l'épicarde. Ce fil, relié à un stimulateur en sentinelle, permettra l'installation sans danger du cathéter. Si l'on juge indispensable d'installer ce moniteur avant l'induction de l'anesthésie, un cathéter-électrode et un pacemaker externe devraient être à portée de la main. La figure 1 illustre un cas vécu.

\section{MEDICATION}

En principe, il est préférable de continuer jusqu'à la chirurgie la médication qui maintient un malade en état de stabilité cardio-vasculaire, 


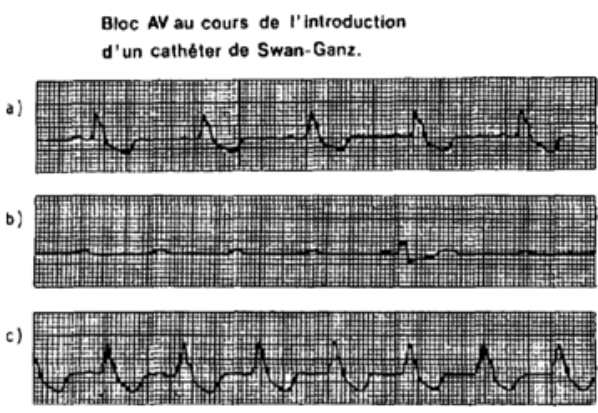

Figure 1 Survenue d'un bloc A-V (b) complet chez une porteuse de (a) bloc de branche gauche, au cours de l'introduction d'un cathéter de Swan-Ganz par la jugulaire interne droite. On a pu corriger rapidement la situation (c) en retirant le Swan-Ganz et en le remplaçant par un cathéter à électrode. Le rythme sinusal est réapparu deux jours après la chirurgie et le malade a récupéré sans problème.

qu'il s'agisse de digitale, de bêta-bloquants, d'anti-arythmiques, de diurétiques ou d'hypotenseurs.

\section{Contact AVEc le Patient}

\section{Rassurer et renseigner}

En principe, l'anesthésiste assigné à un patient voit lui-même son malade la veille de l'intervention. Devant la chirurgie cardiaque, beaucoup de malades sont anxieux, ont peur de souffrir ou de mourir. L'établissement d'un climat de confiance entre le malade et ceux qui participent à son intervention contribue à le rassurer.

Le temps que l'on prend à donner des explications à un malade sur ce qui l'attend au cours des prochains jours et ce qu'on attend de lui comme collaboration, loin de l'effrayer, contribue à le sécuriser. Si, par exemple, on dit à un patient que son intervention se terminera au début de l'après-midi, qu'il passera ensuite de 24 à 36 heures aux soins intensifs, qu'il s'éveillera avec un tube dans la bouche, que ce tube sera un peu inconfortable mais qu'il est placé pour être sûr qu'il respirera bien, qu'il ne sera pas nauséeux mais ne pourra pas parler, que le tube sera enlevé après quelques heures ${ }^{20}$ ou le lendemain matin, qu'il recevra des calmants, qu'un malade sur deux ne se rappelle de rien de la journée de l'opération et de la première nuit, ${ }^{21}$ etc, il réalisera sans que nous ayons à le mentionner (après notre départ, souvent) que nous prenons sa survie pour acquise puisque nous avons pris la peine de lui expliquer tout ce qui précède.

\section{Test d'Allen}

Il ne faut pas prendre pour acquise la perméabilité des arcades palmaires. Des variations anatomiques, l'artériosclérose, ou des embolies, peuvent s'y retrouver. Aussi, avant de canuler une artère radiale, le test d'Allen doit toujours être effectué. (Le Dr. Allen était un interniste de la Clinique Mayo qui a décrit sa méthode en 1929.)

Un test d'Allen modifié est effectué sur les deux mains lors de la visite pré-opératoire. Nous procédons ainsi:

L'artère radiale et la cubitale sont comprimées simultanément avec les doigts pendant que le sujet ferme et ouvre la main deux ou trois fois, jusqu'à ce qu'elle blanchisse. La cubitale est alors relâchée. La main devrait se recolorer en dedans de 15 secondes. Nous ne canulons pas la radiale si la main ne se recolore pas en dedans de 15 secondes. La pédieuse ou la fémorale (comme troisième alternative) sont utilisées dans ces cas.

Le résultat du test d'Allen est ainsi exprimé sur notre feuille de visite opératoire:

$\mathrm{N}$; si la main se recolore en moins de 15 secondes;

$15^{\prime \prime}, 20^{\prime \prime}$ ou $30^{\prime \prime}$ si la recoloration prend 15,20 ou 30 secondes à s'effectuer.

En cas de doute, on peut contrôler le test d'Allen au moyen d'un moniteur de pouls. Il suffit de placer la cellule photo-électrique sur le pouce ou l'index. Dès que la cellule commence à signaler les pulsations, on comprime l'artère radiale. Le maintien du signal, ou son retour après un arrêt de quelques secondes, confirme la perméabilité de l'arcade palmaire.

\section{Allergies}

Dans notre milieu, c'est l'anesthésiste qui, au moment de l'induction de l'anesthésie, a la responsibilité d'administrer par voie intraveineuse les antibiotiques donnés préventivement. Il est donc important de s'informer des allergies du malade, en particulier aux antibiotiques.

\section{Premedication}

Nous utilisons depuis plusieurs années une échelle-maison ${ }^{21}$ pour quantifier l'effet sédatif de la prémédication à l'arrivée du malade au bloc opératoire (tableau V).

Une sédation lourde (score de 2 ou 3) est recherchée. A cette fin, nous utilisons:

\section{1" Chez l'adulte}

Au coucher. Même médication si le patient reçoit 
TABLEAU $V$

EFFETS SEDATIFS DE LA PREMEDICATION

\begin{aligned} & \hline Score \\ &-2 Terrifié - Hystérique - Pleurs - Cris \\ &-1 Inquiet - Anxieux \\ & 0 Pas d'effet apparent \\ & 1 Calme \\ & 2 Somnolent \\ & 3 Endormi - Répond aux questions - S'éveille \\ & si stimulé \\ & 4 Endormi - Ne s'éveille pas à la stimulation \\ & 5 Dépression respiratoire ou circulatoire signi- \\ & ficative \\ & \hline\end{aligned}

déjà un hypnotique et s'en dit satisfait, parfois rien du tout s'il n'en veut pas.

Ou:

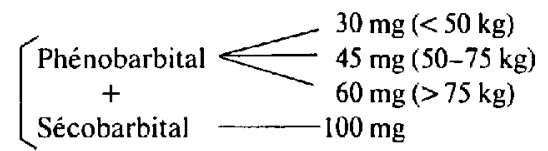

Cette combinaison est très efficace

Le lendemain. A l'éveil, ou au plus tard à 6 heures a.m.: Hydroxyzine $50 \mathrm{mg}$ (parfois $25 \mathrm{mg}$ chez les valvulaires)

A 7 heures a.m., si opéré à 8 heures (à l'appel si opéré plus tard):

$$
\left[\begin{array}{l}
\text { Pentobarbital } \\
2 \mathrm{mg} / \mathrm{kg} \\
+
\end{array} \begin{array}{l}
\text { ad } 150 \mathrm{mg} \text { - total } \\
\text { Scopolamine } 100 \mathrm{mg} \text { - total } \\
0.5 \mathrm{mg}
\end{array} \quad \begin{array}{l}
\text { pour les valvulaires } \\
\end{array}\right.
$$

Certains membres du groupe préfèrent la morphine au pentobarbital et l'administrent à la dose de $0.1 \mathrm{mg} / \mathrm{kg}$ :

moins 25 pour cent si malade âgé

plus 25 pour cent si malade anxieux

2" Chezl'enfant

Hydroxizyne (sirop):

10-20 mg à 6 heures a.m.

$+$

Pentobarbital 3-4 mg/kg i.m. à

Scopolamine: $0.2-0.3 \mathrm{mg}>7$ heures a.m.

\section{REFERENCES}

1. Feigal, D. W. \& Blaisdell. F.W. The estimation of surgical risk. Med. Cl. N.A. 63: 1131-1143 (1979).

2. Robbins, J.A. \& Mushlin, A.I. Preoperative evaluation of the healthy patient. Med. Cl. N.A. 63: 1145-1156(1979)

3. MAILle, J.G. Communication personnelle.

4. Goldman, L., Caloera, D.L., Nussbaum, S.R. Southwick, F.S., Krogstad, D., Murray, B., Burke, D.S., O'Malley, T.A., Goroll, A.H. Caplan, C.H., Nolan, J., Carabello, B. \& SLATER, E.E. Multifactorial index of cardiac risk in non-cardiac surgical procedures. N. Engl. J. Med. 297: 845-850 (1977).

5. Du Cailar, C., Chaitman, B.R. \& Castonguay, $Y$. Risks and benefits of aortocoronary bypass surgery in patients aged 65 years or more. Can. Med. Assoc. J. 122: 771-774 (1980).

6. Vaughan, R. W. Anesthetic management of the obese patient. Weekly Anesthesiology Update, vol. 1, lesson 11 (1978).

7. Waters, D.D., Theroux, P., Halphen, C. \& Mizgala, H.F. Clinical predictors of angina following myocardial infarction. Am. J. Med. 66: 991-996(1979).

8. Pelletier, C., Cossette, R., Dontigny, L. Mercier, C. Page, A.\& Verdant, A. Determinants of mortality following coronary bypass surgery. Can. J. Surg. 23: 199-203 (1980).

9. Golish, J.A. Ahmad, M. \& Yarnal. J.R. Practical application of the flow-volume loop. Cleveland Clin. Quarterly 47: 39-45 (1980).

10. Giesecke, A.H. Diabetes mellitus and the anesthesiologist. Audio-Digest Foundation, vol. 20, California Medical Association (1978).

11. Rossin, A.A. Perioperative care of the diabetic patient. Weekly Anesthesiology Update, vol. 3, lesson 6(1980).

12. SPRITZ, N. Nerve disease in diabetes mellitus. Med. Cl. N.A. 62: 787-798 (1979).

13. Galloway. J.A. \& Bressler, R. Insulin treatment in diabetes. Med. Cl. N.A. 62: 663-680 (1979).

14. Sperling, M.A. Diabetes mellitus in childhood and adolescence. Current Therapy, pp. 405 (1979).

15. White, V.A. \& Kumagai, L.F. Preoperative endocrine and metabolic considerations. Med. $\mathrm{Cl}$. N.A. 63: 1321-1334 (1979)

16. Abrams, L.M \& Chambers, D.A. Preoperative management. In Cardiac Anesthesia. Edited by Kaplan, J.A. New York: Grune \& Stratton, pp. 169-195 (1979)

17. Alberti, K.G.M.M. \& Thomas, D.J.B. The management of diabetes during surgery. Brit. J. Anaesth. 5/: 693-710 (1979).

18. Burke, G.R. \& Gulyassy, P.F. Surgery in the patient with renal disease and related electrolyte disorders. Med. Cl. N.A. 63: 1191-1203 (1979).

19. Thomson, I.R., DalTon, B.C. LapPas, D.G. \& LOWENSTEIN, E. Right bundle-branch block and complete heart block caused by the Swan-Ganz catheter. Anesthesiology 5l: 359-362 (1979).

20. Paiement, B., Boulanger, M., Talllefer, J. \& MAILLE, J.G. Anesthésie el cardiopathie: évolution des concepts et perspectives. Union Méd Can 108: 1158-1161 (1979).

21. Paiement, B., Boulanger, M., Jones, C.W. \& Roy, M. Intubation and other experiences in cardiac surgery: the consumer's views. Canad. Anaesth. Soc. J. 26: 173-180 (1979). 


\section{ABSTRACT}

This text is intended for new residents in the department of anaesthesia of the Montreal Heart Institute. It presents a classification of the risk of cardiovascular surgery used in that institution and discusses current problems encountered with this type of patient (pulmonary and coagulation problems, diabetes renal failure). The attitudes of anaesthetists of this institution towards patients' medication and premedication are also discussed. The risk is classified as usual, increased or high, depending on the presence (or absence) of several factors known to increase the risk: ventricular dysfunction, heart failure, unstable angina or recent infarction. significant involvement of other systems (unstable diabetes, renal insufficiency, significant pulmonary dysfunction), age, emergency surgery and non-cardiac surgery in the presence of important cardiac pathology. With surgical procedures carrying a high mortality, for example dissecting thoracic aneurysm, the usual risk is high and is classified as such. A table of the usual risk of current surgical procedures is proposed. 